\title{
EVALUACIÓN DEL IMPACTO DE LA MODALIDAD FORMATIVA LEARNING BY DOING EN PROFESIONALES SANITARIOS EN ARAGÓN
}

\author{
María Luisa Gracia-Pérez \\ Marta Gil-Lacruz \\ Ana Isabel Gil-Lacruz \\ Universidad de Zaragoza
}

RESUMEN: Los profesionales sanitarios requieren de un proceso formativo constante que les permita hacer frente a retos actuales como: la innovación tecnológica, los nuevos modelos organizacionales, la medicina basada en la evidencia, los cambios epidemiológicos, la participación del usuario, etc. A pesar de los recursos y esfuerzos invertidos, no se ha prestado suficiente atención a la evaluación de la formación de dichos profesionales. Este artículo analiza la evaluación de impacto formativo en base a un programa learning by doing llevado a cabo en el Servicio Aragonés de Salud. En este programa las actividades formativas son propuestas, implementadas y valoradas por los profesionales del servicio en el lugar de trabajo tras un proceso gerencial de selección. Se ha trabajado con una encuesta diseñada de elaboración propia y remitida a los profesionales participantes como alumnos. 182 médicos y enfermeras respondieron on line. Mediante análisis bivariante y regresiones se ha analizado la interacción entre el aprendizaje de conocimientos y habilidades, su trasferencia al lugar del trabajo y la calidad asistencial. En un 94,4\% de los encuestados se constata la relación entre la mejora de la competencia profesional (adquisición de conocimientos y habilidades, utilidad para la profesión, aplicación de los conocimientos nuevos y habilidades en el centro de trabajo, formación en cascada y mejora de los conocimientos y las habilidades) y mejora de la calidad asistencial (medida mediante los indicadores del European Foundation for Quality Management). El diseño de la evaluación del impacto formativo debería estar vinculado a las evaluaciones previas y, sus resultados miden los efectos mediatos en la organización. Esta información se convierte en una herramienta clave para valorar la efectividad del programa ejecutado y para la programación de actuaciones futuras, que garanticen la formación continua de los profesionales sanitarios y permitan mejorar la calidad de sus actuaciones.

PALABRAS CLAVE: Evaluación del impacto formativo, aprender haciendo, formación sanitaria, competencias sanitarias, calidad asistencial sanitaria. 


\title{
FORMATIVE IMPACT ASSESSMENT OF LEARNING BY DOING PROGRAM WITH HEALTH CARE PROFESSIONALS FROM ARAGÓN
}

\begin{abstract}
Health professionals require constant learning process that allows them to address current challenges such as technological innovation, organizational changes, the evidence-based medicine, epidemiological changes, participation, etc. Despite the resources and efforts invested, it has not paid enough attention to assessing the training of these professionals. This article analyzes the formative impact assessment based on a learning by doing program conducted in the Aragon Health Service. The training activities were proposed, implemented and evaluated by service professionals in the workplace after a management selection process. We have worked with ad hoc survey designed and sent to participating professionals and students. 182 doctors and nurses responded online. By bivariate regression analysis and analyzed the interaction between learning knowledge and skills, their transfer to the workplace and the quality of care. $94.4 \%$ of respondents the relationship between improving professional skills (acquisition of knowledge and skills useful to the profession, application of new knowledge and skills in the workplace, training cascading notes and improving knowledge and skills) and improved quality of care (measured by indicators of European Foundation for Quality Management). The design of the impact evaluation of the training should be linked to previous assessments and measure results consequential effects on the organization. This information becomes a key tool to assess the effectiveness of the executed program and programming future actions, to ensure the continued training of health professionals and to improve the quality of their performances.
\end{abstract}

KEYWORDS: Educative impact assessment, learning by doing, health professional's education, medical skills, health care quality.

Recibido: 15/11/2015

Aceptado: 02/01/2016

Correspondencia: Marta Gil Lacruz, Universidad de Zaragoza, Facultad de Ciencias de la Salud, C/ Domingo Miral s/n, 50009 Zaragoza. Email: mglacruz@unizar.es.

\section{INTRODUCCIÓN}

El desarrollo de la formación continua como política estratégica de las organizaciones, requiere de un proceso de investigación riguroso que abarca tres fases principales (Gracia, 2015):

- La planificación previa en base a la detección y priorización de necesidades que comparten los profesionales objeto de ese aprendizaje (Bausela, 2004; Oliver, 2002). En el entorno sanitario éstas pueden hacer referencia a la nueva gobernanza, innovaciones tecnológicas, pautas epidemiológicas, etc. 
- La ejecución que implica la selección de una metodología adecuada para la transmisión de los nuevos conocimientos y habilidades. En esta investigación, se ha seleccionado la metodología "learning by doing" por su capacidad de transferencia de los contenidos aprendidos al puesto de trabajo, únicamente por debajo de la instrucción individual y muy por encima de las practicas docentes tradicionales (Fernández, Porcel, Nuviala, Pérez, Tamayo, Grao y González, 2012). Frente a otras modalidades el alumno adquiere un rol de agente activo a lo largo de la intervención formativa, necesario si se desea mejorar su práctica clínica.

- La evaluación del proceso, que a menudo se centra en conocer los efectos inmediatos que produce la intervención formativa: la satisfacción y el aprendizaje de los participantes. Sin embargo, si no se exploran los efectos a largo plazo, ej. en la calidad asistencial del sistema sanitario, la intervención evaluativa queda inacabada porque no permite inferir la utilidad y alcance de la formación (Biencinto y Carballo, 2006).

En relación a los efectos de la formación, Ubillos y Golburu (2010) distinguen entre evaluación de resultados y evaluación de impacto. La primera se ocupa de conocer los efectos de la implementación de la acción formativa a corto plazo y que afecta a los participantes de la formación, mientras que la segunda estudia las consecuencias a largo plazo y los cambios que genera en el entorno donde el profesional, que ha participado en el programa se desarrolla laboralmente, determinando la efectividad real de la actuación formativa. Soto (2011) reivindica el valor de este impacto porque no solo garantiza su sostenibilidad ante cambios de autoridades o crisis económicas sino que también fortalece el apoyo de todos los implicados, ya sean participantes o responsables de los servicios, departamentos o de la propia organización.

Uno de los autores que aporta una definición interesante del término impacto es Pineda (2000): "Las repercusiones que la realización de unas acciones formativas conllevan para la organización, en términos de respuesta a las necesidades de formación, de resolución de problemas y de contribución al alcance de los objetivos estratégicos que la organización tiene planteados" (p. 124). De manera complementaria, Fernández (2006) entiende la evaluación de impacto como: "Un proceso orientado a medir los resultados generados (cambios y causas) por las acciones formativas desarrolladas en el escenario socioprofesional originario de las mismas al cabo del tiempo" (p. 20).

En la práctica organizativa, el ejercicio de la evaluación de impacto está poco extendido. Las razones apuntan a varias cuestiones como: la complejidad metodológica que acompaña al proceso, la falta de flexibilidad e información de la propia estructura institucional, la escasez de políticas que desarrollen la evaluación, etc. (Onrubia, 2005; Tejada y Fernández, 2007). De donde se infiere, una ausencia relevante de la extensión de la cultura evaluativa en la organización, y en concreto, en la Administración Pública, pese a los esfuerzos, entre otras instituciones de la Agencia Estatal de Evaluación de las Políticas Públicas y la Calidad de los Servicios (AEVAL, 2010). Dicha agencia defiende los planteamientos del new public management como herramienta de mejora en el proceso de la evaluación, entendida no 
solo como un instrumento de gestión, sino como la respuesta a un nuevo modelo de democracia apoyado en los valores de responsabilidad, transparencia, participación y rendición de cuentas.

La extensión de la cultura evaluativa junto con el public management, facilitaría la evaluación del impacto de la formación al permitir verificar el proceso de consecución de los objetivos y mejorar dicho proceso (Pineda, 2000). Este tipo de evaluación conlleva un acto de perfeccionamiento de la formación y de la propia metodología evaluativa. Supone trabajar sobre el principio de evidencia. Presenta por tanto, dos finalidades: gerencial y política (Merino, 2007).

A pesar de que no se dispone de un único modelo evaluativo, la mayoría de ellos coincide en resaltar su carácter complejo, multidisciplinar, dinámico y de trabajo en equipo. En esta investigación y desde un marco sistémico integrador, se han seleccionado las siguientes propuestas afines al proceso formativo realizado:

El modelo holístico / sistémico aporta una visión organizativa de la formación y la evaluación. Surge como modelo basado en el sistema frente a los modelos basados en los resultados (ej. Tyler, 1942), con la intención de abordar parámetros que éste no considera, tales como el contexto cultural de la organización. Define el programa formativo como parte de las políticas de recursos humanos (Eseryel, 2002). La evaluación implica el compromiso de todos y cada uno de los agentes, para averiguar en qué términos la formación ha conseguido sus objetivos de mejora y afrontar cambios contextuales ya sean de orden clínico, asistencial, tecnológico o de otra índole (Cabrera, 2003). A diferencia de otros modelos, en los que la evaluación se realiza a corto plazo, ej. Kirkpatrick (2003), el modelo sistémico le concede gran importancia al proceso de evaluación y al tiempo que media entre la formación y la evaluación (Gracia, 2015).

El modelo de evaluación por procesos complementarios (CIPP, Stufflebeam y Shinkfield, 1987) constituye una variante que se integra dentro de los modelos sistémicos. El modelo CIPP hace hincapié en los inputs (agentes, modalidad formativa, recursos), los procesos (planificación, selección de alumnos, docentes y centros, ejecución del aprendizaje, etc.) y los productos clasificados en inmediatos y mediatos. Prioriza la necesidad de integración y coherencia de las políticas de recursos humanos y considera la formación como objetivo estratégico de las organizaciones (Capapé, Susaeta, Pin, Gallifa y García, 2011; Peña y Hernández, 2007).

Según el modelo de Moore (2009) (Return on Strategy) la acción formativa se dirige al conjunto global de la organización con el fin de medir su impacto integral. Para su logro es preciso establecer en la planificación del programa, la metodología de evaluación de impacto, en la que se definan las secuencias del proceso y los agentes implicados.

Guerra-López (2007) (Proceso para evaluar impacto) desde una perspectiva sistémica, propone el estudio de la interdependencia de factores organizativos. Define tres tipos de resultados que se deberían obtener con la evaluación del impacto formativo: Resultados estratégicos (resultados en la organización a largo plazo que benefician a la sociedad); Resultados tácitos (operaciones y procesos 
que servirán para alcanzar los resultados inmediatos); Resultados operacionales (el conjunto de la información obtenida sobre la organización que permitirá valorar las intervenciones).

Modelo "Success Case Method" (SCM) de Brikerhoff (1981). De acuerdo con Rutty (2007), lo característico del modelo es el detalle con el que se estudia la fase de la planificación y del proceso del aprendizaje. La propuesta del método del caso exitoso se desarrolla a través de la detección de casos extremos: casos de participantes que han transferido su formación al puesto de trabajo y participantes que no. En la comparación se pretenden detectar cuáles son los factores que dieron lugar al éxito y los obstáculos con los que se encontraron los participantes que no aplicaron el aprendizaje.

Al estudiar estos modelos, diversos autores coinciden en la selección de las principales dimensiones de la evaluación formativa: la satisfacción del participante, el logro de los objetivos de aprendizaje por los participantes, la coherencia pedagógica del proceso de formación, la transferencia de los aprendizajes al puesto de trabajo, el impacto de la formación en los objetivos de la organización, la empleabilidad (evaluación de conocimientos, habilidades y actitudes), y el valor añadido en el contexto organizativo (Chacón, Holgado, López y Sanduvete, 2006; Pineda 2000).

El estudio de estas dimensiones permite establecer los efectos formativos inmediatos (incremento de conocimientos y habilidades, actitudes, valores, intereses) y mediatos o diferidos (creatividad, mejora del estatus profesional y mejora organizacional, desarrollo económico) (Biecinto y Carballo, 2006).

Cada una de estas dimensiones, requiere una valoración propia e interrelacionada con las demás categorías. Por ejemplo, la satisfacción del estudiante con la actividad formativa es una de las dimensiones más estudiadas en la literatura de la evaluación de impacto. A propósito de su estudio, Granado, Puig y Aguilar (2012) plantean la sutil distinción entre las sensaciones positivas de agrado, entretenimiento, etc. que la formación proporciona al participante y que presenta una influencia mínima con la transferencia y la satisfacción vinculada a la utilidad percibida de los programas formativos para responder a las necesidades del puesto de trabajo, que en este caso si se relaciona directamente con la mejora de competencias.

La adquisición o mejora de la competencia profesional se inicia con "saber", es decir con el aprendizaje de nuevos conocimientos, habilidades y destrezas y finaliza con el "demostrar" (Miller, 1990). La competencia profesional se demuestra con la aplicación de lo aprendido en el puesto de trabajo, redundando en una mejora organizativa y en una mejora de la calidad asistencial (Méndez, 2007). Por tal motivo en esta investigación, previo a definir la mejora de la calidad asistencial como variable dependiente del estudio, interesa conocer en qué medida se relaciona por separado la mejora de conocimientos y habilidades y la aplicación de conocimientos con el resto de las variables (Tejeda, 2011).

En el ámbito clínico, en concreto en el Servicio Aragonés de Salud, los profesionales sanitarios trabajan con los criterios basados en el modelo de evaluación de calidad EFQM (European Foundation for Quality Management). Entre los criterios 
que define el modelo se encuentran: liderazgo, personas, estrategias, resultados en pacientes, resultados en sociedad. Estos criterios valoran las acciones formativas haciendo hincapié en la calidad de los procesos dentro del contexto sanitario.

Además, la organización sanitaria tiende a basar su actuación en protocolos estandarizados, auditorías externas e indicadores de desempeño que confieren una gran precisión a los procesos de diagnóstico, cura y rehabilitación (Green, Durand, Hutchings y Black, 2011).

Estas características hacen que dicho entorno sea un ámbito receptivo para la inclusión de procedimientos de evaluación del impacto formativo en experiencias learning by doing. Este articulo integra las aportaciones de los diferentes modelos de evaluación de impacto formativo, en relación a la experiencia learning by doing en un contexto sanitario aragonés.

\section{Método}

Esta investigación analiza un modelo de evaluación de impacto de la formación en la organización sanitaria, una vez que ha sido transferida al puesto de trabajo de quien se ha formado. Plantea la cuestión de si el programa learning by doing, focuss, orientado a la mejora de la calidad asistencial, ejerce influencia en el profesional y en la organización sanitaria, dando lugar a resultados concretos.

Las variables dependientes de la formación y diana de la evaluación de impacto son: la mejora de los conocimientos y habilidades, la aplicación de conocimientos y habilidades y la mejora la calidad asistencial. Interesa analizar que variables presentan un mayor peso en la valoración de la calidad asistencial.

\section{UNIVERSO Y MUESTRA}

Como base aplicada para la consecución de estos objetivos se utilizará el programa de formación continua focuss desarrollado por el Instituto Aragonés de Ciencias de la Salud (IACS) y dirigido al personal sanitario del Servicio Aragonés de Salud (SALUD). Su naturaleza interactiva y dialéctica lo distingue de otros programas formativos por el hecho de que los profesionales sanitarios son los docentes que proponen cursos y también los estudiantes que participan en las actividades seleccionadas. Son profesionales de la misma organización y comparten la misma cultura clínica e institucional. Las actividades formativas se desarrollan dentro de su entorno profesional. Son los profesionales los que proponen las actuaciones formativas en las que van a ser los docentes y responsables. Dichas propuestas serán analizadas y seleccionadas por el comité de coordinación. A su vez, este comité está compuesto por profesionales del Instituto Aragonés de Ciencias de la Salud, (Servicio Aragonés de Salud). Los profesionales-alumnos solicitan las actividades formativas y son seleccionados en función de la adecuación de su perfil profesional al programa formativo.

Este programa ha sido seleccionado para analizar la evaluación de su impacto formativo porque sus objetivos son coherentes con el desarrollo de la organización. Con este fin, pretende crear redes informales de cooperación entre centros y ser- 
vicios, mejorar la satisfacción de los profesionales, su capital humano y la calidad asistencial. Además su metodología learning by doing, facilita la transferencia de conocimientos y habilidades adquiridos tras la formación al puesto de trabajo.

El programa basado en el aprendizaje de técnicas diagnósticas, quirúrgicas y terapéuticas, se inició en el 2005. Ha crecido de manera exponencial e implica un proceso selectivo importante tanto de propuestas formativas presentadas como de posibles estudiantes. En esta investigación, los datos se corresponden con la convocatoria 2010. Se aceptaron un total de 77 actuaciones (2.500 horas) a ejecutarse en 66 centros docentes, y a través de 394 profesionales sanitarios que ejercieron de docentes. La temática de dichos cursos fue muy variada ej. valoración geriátrica, enfermería en urgencias de pediatría, tratamiento del dolor, diagnóstico microbiológico de enfermedades infecciosas, cirugía: laparoscopia avanzada. Independientemente de las características específicas del tema, cada curso presentó un promedio de asistencia de 5 estudiantes y 31 horas formativas distribuidas a lo largo de una semana.

Los 385 alumnos profesionales que participaron en los cursos durante la convocatoria del 2010 se corresponden con el universo de esta investigación. 222 profesionales ejercen su labor en centros de atención primaria (57,66\%), 118 en atención especializada (30,65\%) y 45 en servicios de emergencias (11,69\%). Aunque el programa es multidisciplinar, destaca la participación de médicos y personal de enfermería (94,54\% del universo) procedentes de centros sanitarios urbanos (ej. hospitales, centros de salud). Un 73,25\% de los alumnos fueron mujeres.

El instrumento on line se envió a las direcciones electrónicas de todos los alumnos cuatro meses después de haber terminado su formación. En el correo se explicaba el anonimato de respuesta, el motivo y la importancia de su participación y se determinaba un plazo de diez días para su remisión.

Se recibieron 182 cuestionarios realizados de forma correcta, que integran la muestra de este artículo. Esta muestra implica el $47,27 \%$ del universo. Se trata de un muestreo intencional no aleatorio. La modalidad de presentación on line pudo influir en el absentismo de respuesta. No obstante, la muestra refleja las tendencias encontradas en el universo de estudiantes del programa.

La edad media de los participantes fue de 44,41 años (IC95\%: 43,17-45,66). En cuanto al sexo, predominan las mujeres profesionales $(78,8 \%)$ frente a sus compañeros varones $(21,2 \%)$. Contestaron 77 médicos $(42,78 \%$ de la muestra) y 105 personal de enfermería $(57,07 \%)$. Las áreas de trabajo más comunes fueron: atención primaria en 103 profesionales (56,6\%), atención especializada en $54(29,7 \%)$ y emergencias en 25 estudiantes (13,7\%). El valor promedio de los años trabajados en el Servicio Aragonés de Salud fue de 17,54 años (IC95\%: 16,24-18,83).

En cada uno de los cursos, se efectúan tres evaluaciones a lo largo del proceso formativo. En la primera evaluación, anterior a la ejecución de las actividades formativas, el equipo coordinador analiza las propuestas formativas recibidas de los profesionales docentes. El equipo coordinador valora las actividades formativas en cuanto a: contenidos, adecuación de los perfiles de profesionales y centros sanitarios donde se Ilevaran a cabo. El objeto de este estudio previo es priorizar la relevancia, pertinencia e idoneidad de las propuestas con el fin de planificar el programa. 
La segunda valoración, se realiza inmediatamente después de terminar la acción formativa por parte de los alumnos. En esta evaluación se tiene en cuenta los efectos inmediatos del curso en términos de satisfacción y aprendizaje. Dichas dimensiones valoradas por los estudiantes hacen referencia a los siguientes criterios: contenidos, metodología, actitud y aptitud del docente, gestión del curso, instalaciones, etc.

En la tercera evaluación se vuelve a recurrir al alumno para recabar la información sobre los efectos mediatos de la intervención. Se entiende por efecto mediato la posible variación en la práctica profesional del estudiante y por consiguiente, en su centro de trabajo. Este artículo se basa en la información obtenida en esta tercera fase al hacer referencia directamente al impacto formativo.

\section{INSTRUMENTO}

Como objeto de evaluación se definen un conjunto de indicadores y variables que comprenden las principales dimensiones de la organización y de la intervención formativa, siguiendo los modelos sistémicos de evaluación de impacto:

- Modalidad formativa. Contempla dos variables: a) las preferencias de la muestra en relación a la modalidad learning by doing, en comparación con experiencias formativas previas, ej. clases magistrales, talleres, conferencias, etc. Adopta dos posibles respuestas 0 (no), 1 (si). b) nivel de retención de conocimientos / habilidades como indicador de la capacidad de transferir esta información al puesto de trabajo. Se operativiza en cuatro categorías, siendo 0 (ninguno), el nivel más bajo de retención y 3 (mucho), el más alto.

- Satisfacción del alumno y aprendizaje. Como variables diana esta dimensión integra la medida autorreporte de satisfacción con: a) el docente; b) los contenidos del programa; c) la metodología del mismo; d) el tiempo invertido y e) la gestión de la organización institucional. Para la operativización de esta dimensión a las cinco variables se les ha asignado un valor dicotómico. 0 (no), 1 (si).

- Mejora de la competencia profesional. Mediante una escala tipo Likert que oscila entre 0 (ninguno) y 3 (mucho) se han analizado las siguientes variables (Miller, 1990): a) adquisición de nuevos conocimientos / habilidades; b) utilidad del programa para su profesión; c) aplicación de conocimientos / habilidades; d) mejora de los conocimientos / habilidades en el puesto de trabajo; e) formación en cascada (transmisión de conocimientos y habilidades a otros compañeros.

- Mejora de la calidad asistencial. Referida a la percepción que el profesional tiene tras su formación, en términos de mejora en las técnicas diagnósticas, de tratamiento y de recuperación. La variable presenta dos categorías 0 (no), 1 (si).

- Repercusión económica. Integra dos variables: a) Optimización de recursos. Se refiere al juicio de los profesionales sobre si la aplicación del aprendizaje ha servido para el mejor aprovechamiento de los recursos del centro o servicio. Adopta dos posibles valores 0 (no), 1 (si). b) Influencia en el gasto. Refleja 
la opinión de los participantes acerca de la influencia que la transferencia del aprendizaje ha ejercido en el gasto del centro/servicio. Esta variable presenta las siguientes categorías: 0 (ha aumentado el gasto), 1 (no ha influido en el gasto), 2 (ha reducido el gasto).

Estas variables y dimensiones se integran en un cuestionario de elaboración propia compuesto de 15 ítems. A las preguntas relativas a las dimensiones evaluativas se añadió un bloque de preguntas sociodemográficas (edad, sexo, categoría profesional, años trabajados en el puesto de trabajo, número de actividades formativas realizadas en el programa).

La codificación y análisis de datos se realizó con los programas estadísticos SPSS v.15 (licencia de la Universidad de Zaragoza) y Epidat 4.0. Para la comprobación de la validez del mismo se realizó un estudio piloto sobre una muestra de 10 personas seleccionadas al azar (5 personal médico y 5 profesionales de la enfermería, colaboradoras del IACS), quienes una vez revisado el cuestionario estimaron correctas la selección de dimensiones, variables y categorías).

\section{Resultados}

\section{Resultados descriptivos}

A continuación se describen los resultados principales obtenidos desagregados por variables y dimensiones en su conjunto:

- Modalidad formativa. En cuanto a las preferencias de la muestra en relación a la modalidad learning by doing sobre otros métodos, el 100\% reconoce sus efectos positivos. El 70,1\% manifiesta haber aprendido bastantes conocimientos y el 20,4\% mucho. La variable suma de las dos anteriores que se define como resumen de la dimensión adopta valores entre 0 y 4 . Presenta una media de 3,11 y una desviación estándar de 0,54.

- Satisfacción del alumno y aprendizaje. Los valores de satisfacción fueron elevados en todas las variables de la dimensión satisfacción: con el docente $(93,8 \%)$, con los contenidos del programa $(94,4 \%)$, la metodología utilizada $(96,2 \%)$, el tiempo invertido $(87,1 \%)$, la gestión del IACS $(95,1 \%)$. A fin de obtener un valor general de la dimensión satisfacción se ha calculado la integración de las cinco variables. Al ser la suma de cinco variables con posibles valores 0 (no), 1 (si), podrá tomar valores entre 0 y 5 siendo la satisfacción nula $=0$ y la satisfacción total $=5$. La media de la satisfacción es de 4,67 con una desviación estándar de 0,82.

- Mejora de la competencia profesional: a) adquisición de nuevos conocimientos / habilidades: un 59,7\% de la muestra adquirió muchos, y bastantes un $35,2 \%$ b) utilidad del programa para su profesión: un $99,5 \%$ de la muestra valoró el programa como bastante y muy útil; c) aplicación de conocimientos / habilidades. Solo un $13,2 \%$ de la muestra manifestó no tener la oportunidad de aplicarlos. Las razones aducidas en la pregunta abierta se refieren a falta de pacientes, equipamiento, tiempo, etc. d) mejora de los co- 
nocimientos / habilidades en el puesto de trabajo. Un 91,6\% de la muestra coincide en resaltar esta mejora e) formación en cascada. Un 79,6\% de la muestra (transmisión de conocimientos y habilidades a otros compañeros. La variable suma de las cinco anteriores, como resumen de la dimensión mejora de la competencia profesional por el tipo de variables incluidas toma valores entre 0 y 9 , siendo 9 el valor que indica mayor percepción de la competencia profesional. La muestra presenta una media de 7,49 y una desviación estándar de 1,31.

- Mejora de la calidad asistencial. El 95,7\% de los profesionales encuestados coinciden en valorar positivamente como la formación recibida influye en la mejora de las técnicas diagnósticas, de tratamiento y de recuperación que aplican.

- Repercusión económica. A la pregunta de si los encuestados consideran que la formación realizada ha optimizado la utilización de los recursos de sus centros, un 79\% de los participantes estima que sí. Un 73,7\% de los encuestados valora que la formación aumenta el gasto sanitario. La variable suma de ambas se presenta en una escala del 0 al 3. En la muestra presenta una media de 1,25 y una desviación estándar de 1,11.

En los siguientes análisis estadísticos se consideran como variables dependientes: la mejora de los conocimientos y las habilidades, la aplicación de conocimientos y habilidades y la mejora de la calidad asistencial. Para evitar el solapamiento en las técnicas estadísticas inferenciales, se eliminan de la dimensión competencia profesional, las variables mejora y aplicaciones de conocimientos y habilidades; que se analizan de manera independiente.

\section{Resultados inferenciales en función de las variables}

En primer lugar, se realizó un análisis bivariante para observar las relaciones existentes entre las variables a estudio. Tal y como se expuso en el marco teórico, se parte de la base de que la mejora de los conocimientos y habilidades, resulta un paso previo para su aplicación en el puesto de trabajo (Miller, 1990). Conocimiento y aplicación deberían influir conjuntamente en la mejora de la calidad asistencial (Méndez, 2007; Tejeda, 2011).

a) Análisis bivariante: mejora de conocimientos y habilidades en el puesto de trabajo

Las pruebas estadísticas (ver tabla 1) señalan que la mejora de conocimientos y habilidades se encuentra relacionada con: número de programas realizados en anteriores ediciones, satisfacción con: el profesor, los contenidos del programa, el tiempo invertido, la gestión del IACS, la metodología, la retención y adquisición de nuevos conocimientos, la utilidad del programa para la profesión, la aplicación de conocimientos y habilidades en el centro de trabajo, la formación en cascada, la mejora de la calidad asistencial y la optimización de recursos. 
Tabla 1. Relaciones entre las variables de estudio y mejora de los conocimientos y habilidades

\begin{tabular}{|c|c|c|}
\hline Variables & Prueba estadística & p valor \\
\hline \multicolumn{3}{|l|}{ Perfil socio demográfico } \\
\hline Sexo & Fisher & 1,000 \\
\hline Edad & Fisher & 0,603 \\
\hline Categoría Profesional & Fisher & 0,791 \\
\hline Lugar de trabajo & Chi cuadrado & 0,183 \\
\hline Antigüedad en el trabajo & Fisher & 1,000 \\
\hline$N^{o}$ focuss realizados previamente & Fisher & 0,039 \\
\hline \multicolumn{3}{|l|}{ Satisfacción con } \\
\hline El profesor & Chi cuadrado & 0,016 \\
\hline Los contenidos & Fisher & 0,004 \\
\hline El tiempo & Fisher & 0,019 \\
\hline Gestión del IACS & Fisher & 0,023 \\
\hline Satisfacción metodología & Fisher & $<0,001$ \\
\hline \multicolumn{3}{|l|}{ Modalidad } \\
\hline Modalidad formativa & Fisher & - \\
\hline Retención de conocimientos & Chi cuadrado & 0,004 \\
\hline \multicolumn{3}{|l|}{ Mejora competencia profesional } \\
\hline Adquisición de conocimientos & Chi cuadrado & $<0,001$ \\
\hline Utilidad del programa & Chi cuadrado & 0,001 \\
\hline Aplicación conoc/habilidades & Fisher & $<0,001$ \\
\hline Formación en cascada & Fisher & 0,017 \\
\hline Mejora de la calidad asistencial & Fisher & $<0,001$ \\
\hline \multicolumn{3}{|l|}{ Repercusión económica } \\
\hline Optimización de recursos & Fisher & 0,001 \\
\hline Influencia en el gasto & Chi cuadrado & 0,089 \\
\hline
\end{tabular}

Fuente: Elaboración propia.

b) Análisis bivariante: aplicación de conocimientos y habilidades en el puesto de trabajo

La variable aplicación de conocimientos y habilidades en el puesto de trabajo se relaciona con las siguientes variables: lugar del puesto de trabajo, número de programas realizados en anteriores ediciones, satisfacción con el profesor, los contenidos del programa, el tiempo invertido, la metodología, la adquisición de nuevos conocimientos, la mejora de conocimientos y habilidades, la formación en cascada, la mejora de la calidad asistencial y la optimización de los recursos (ver tabla 2). 
Tabla 2. Relaciones entre las variables de estudio y aplicación de los conocimientos y habilidades

\begin{tabular}{|c|c|c|}
\hline Variables & Prueba estadística & $\mathrm{p}$ valor \\
\hline \multicolumn{3}{|l|}{ Perfil socio demográfico } \\
\hline Sexo & Chi cuadrado & 0,709 \\
\hline Edad & Chi cuadrado & 0,560 \\
\hline Categoría Profesional & Chi cuadrado & 0,609 \\
\hline Lugar de trabajo & Chi cuadrado & 0,004 \\
\hline Antigüedad en el trabajo & Chi cuadrado & 0,958 \\
\hline$N^{o}$ focuss realizados previamente & Fisher & 0,001 \\
\hline \multicolumn{3}{|l|}{ Satisfacción con } \\
\hline El profesor & Chi cuadrado & 0,007 \\
\hline Los contenidos & Fisher & 0,027 \\
\hline El tiempo & Fisher & 0,044 \\
\hline Gestión del IACS & Fisher & 0,295 \\
\hline Satisfacción metodología & Fisher & 0,006 \\
\hline \multicolumn{3}{|l|}{ Modalidad } \\
\hline Modalidad formativa & Fisher & - \\
\hline Retención de conocimientos & Chi cuadrado & 0,355 \\
\hline \multicolumn{3}{|l|}{ Mejora competencia profesional } \\
\hline Adquisición de conocimientos & Chi cuadrado & 0,010 \\
\hline Utilidad del programa & Chi cuadrado & 0,336 \\
\hline Formación en cascada & Fisher & 0,001 \\
\hline Mejora de la calidad asistencial & Fisher & 0,001 \\
\hline \multicolumn{3}{|l|}{ Repercusión económica } \\
\hline Optimización de recursos & Fisher & $<0,001$ \\
\hline Influencia en el gasto & Chi cuadrado & 0,172 \\
\hline
\end{tabular}

Fuente: Elaboración propia.

c) Análisis bivariante: mejora de la calidad asistencial

La mejora de la calidad asistencial se relaciona con la satisfacción con el profesor, los contenidos del programa, el tiempo invertido, la metodología, la retención, adquisición y aplicación de nuevos conocimientos, la mejora de la competencia profesional, la formación en cascada y la optimización de los recursos (ver tabla 3). 
Tabla 3. Relaciones entre las variables de estudio y mejora de la calidad asistencial

\begin{tabular}{|c|c|c|}
\hline Variables & Prueba estadística & p valor \\
\hline \multicolumn{3}{|l|}{ Perfil socio demográfico } \\
\hline Sexo & Fisher & 0,633 \\
\hline Edad & Fisher & 0,713 \\
\hline Categoría Profesional & Fisher & 0,700 \\
\hline Lugar de trabajo & Chi cuadrado & 0,993 \\
\hline Antigüedad en el trabajo & Fisher & 0,712 \\
\hline $\mathrm{N}^{\circ}$ focuss realizados previamente & Fisher & 0,096 \\
\hline \multicolumn{3}{|l|}{ Satisfacción con } \\
\hline El profesor & Chi cuadrado & 0,003 \\
\hline Los contenidos & Fisher & $<0,001$ \\
\hline El tiempo & Fisher & 0,027 \\
\hline Gestión del IACS & Fisher & 0,261 \\
\hline Satisfacción metodología & Fisher & 0,001 \\
\hline \multicolumn{3}{|l|}{ Modalidad } \\
\hline Modalidad formativa & Fisher & - \\
\hline Retención de conocimientos & Chi cuadrado & $<0,001$ \\
\hline \multicolumn{3}{|l|}{ Mejora competencia profesional } \\
\hline Adquisición de conocimientos & Chi cuadrado & $<0,001$ \\
\hline Utilidad del programa & Chi cuadrado & 0,233 \\
\hline Mejora de conocimientos habilidades & Fisher & $<0,001$ \\
\hline Aplicación conoc/habilidades & Fisher & 0,001 \\
\hline Formación en cascada & Fisher & 0,018 \\
\hline \multicolumn{3}{|l|}{ Repercusión económica } \\
\hline Optimización de recursos & Fisher & 0,002 \\
\hline Influencia en el gasto & Chi cuadrado & 0,246 \\
\hline
\end{tabular}

Fuente: Elaboración propia.

\section{Resultados inferenciales en función de las dimensiones}

Una vez analizadas las interacciones entre variables, conviene priorizar dichos resultados en función de las dimensiones que las integran.

La valoración positiva de la formación recibida en cuanto a mejora de los conocimientos y habilidades adquiridos y mejora de la calidad asistencial se relaciona 
de manera estadísticamente significativa con todas las dimensiones: satisfacción, modalidad, mejora de la competencia profesional y repercusión económica (ver tabla 4).

Cuando se trata de aplicar dichos conocimientos, la interacción también es positiva para las dimensiones: satisfacción, mejora de la competencia profesional y repercusión económica.

Tabla 4. Relación entre las dimensiones: mejora y aplicación de conocimientos y habilidades con la mejora de la calidad asistencial, media (DE)

\begin{tabular}{|c|c|c|c|}
\hline Dimensiones y variables & p valor & No hay mejora & Si hay mejora \\
\hline \multicolumn{4}{|l|}{$\begin{array}{c}\text { Dimensión: mejora de conocimientos y } \\
\text { habilidades }\end{array}$} \\
\hline Satisfacción & 0,005 & $3,64(1,87)$ & $4,77(0,55)$ \\
\hline Modalidad & 0,003 & $2,67(0,49)$ & $3,15(0,52)$ \\
\hline Mejora competencia profesional & $<0,001$ & $4,69(0,95)$ & $6,72(1,05)$ \\
\hline Repercusión económica & $<0,001$ & $0,20(0,42)$ & $1,30(1,10)$ \\
\hline \multicolumn{4}{|l|}{$\begin{array}{l}\text { Dimensión: aplicación de conocimientos } \\
\text { y habilidades }\end{array}$} \\
\hline Satisfacción & 0,013 & $4,09(1,41)$ & $4,76(0,64)$ \\
\hline Modalidad & 0,154 & $2,25(0,50)$ & $3,15(0,54)$ \\
\hline Mejora competencia profesional & $<0,001$ & $5,65(1,37)$ & $6,67(1,05)$ \\
\hline Repercusión económica & 0,001 & $0,53(1,01)$ & $1,33(1,10)$ \\
\hline \multicolumn{4}{|l|}{$\begin{array}{c}\text { Dimensión. Mejora de la calidad } \\
\text { asistencial }\end{array}$} \\
\hline Satisfacción & $<0,001$ & $2,83(1,72)$ & $4,75(0,66)$ \\
\hline Modalidad & 0,003 & $2,25(0,50)$ & $3,15(0,54)$ \\
\hline Mejora competencia profesional & 0,007 & $5,50(2,07)$ & $7,70(1,12)$ \\
\hline Repercusión económica & 0,001 & $0,14(0,38)$ & $1,34(1,12)$ \\
\hline
\end{tabular}

Fuente: Elaboración propia.

De los resultados obtenidos se infiere que las relaciones entre las variables mejora y aplicación de conocimientos y mejora de la calidad de vida asistencial, son estadísticamente significativas. El 98\% de los alumnos que creen que han mejorado sus conocimientos y el 92,2\% que los han aplicado al puesto de trabajo, también consideran que ha mejorado la calidad de asistencia a los usuarios (ver tabla 5). 
Tabla 5. Relación entre las variables mejora de conocimientos y habilidad y aplicación por los mismos en función de la mejora de la calidad asistencial

\begin{tabular}{|c|c|c|c|}
\hline & \multicolumn{2}{|c|}{ Mejora de conocimientos y habilidades } & \multirow{2}{*}{ Total } \\
\hline Mejora de la calidad asistencial & No & Si & \\
\hline \multirow[t]{2}{*}{ No } & 4 & 3 & 7 \\
\hline & $28,60 \%$ & $2,00 \%$ & $4,30 \%$ \\
\hline \multirow[t]{2}{*}{ Si } & 10 & 146 & 156 \\
\hline & $71,40 \%$ & $98,00 \%$ & 95,70 \\
\hline \multirow[t]{3}{*}{ Total } & 14 & 1,49 & 163 \\
\hline & $100 \%$ & $100 \%$ & $100 \%$ \\
\hline & \multicolumn{2}{|c|}{$\begin{array}{c}\text { Aplicación de conocimientos y } \\
\text { habilidades }\end{array}$} & \multirow[t]{2}{*}{ Total } \\
\hline Mejora de la calidad asistencial & No & Si & \\
\hline No & 4 & 3 & 7 \\
\hline$\%$ & $40,00 \%$ & $2,00 \%$ & $4,40 \%$ \\
\hline Si & 6 & 147 & 153 \\
\hline$\%$ & $60,00 \%$ & $98,00 \%$ & $95,60 \%$ \\
\hline Total & 10 & 150 & 160 \\
\hline Total \% & $100 \%$ & $100 \%$ & $100 \%$ \\
\hline
\end{tabular}

Fuente: Elaboración propia.

El siguiente análisis pretende formular un modelo predictivo de la variable mejora de la calidad asistencial a partir de modelos de regresión logística (tabla 6) y con los valores unificados para las 4 dimensiones. No se incluye ninguna de las variables de la dimensión socio-laboral por el escaso impacto encontrado en los análisis bivariantes.

Tabla 6. Resultados $O R$ Crudo y OR ajustado de las variables suma y la variable dependiente: mejora de la calidad asistencial

\begin{tabular}{ccccc}
$\begin{array}{c}\text { Variables } \\
\text { independientes }\end{array}$ & $\begin{array}{c}\mathbf{p} \\
\text { valor }\end{array}$ & OR CRUDO (IC 95\%) & $\begin{array}{c}\mathbf{p} \\
\text { valor }\end{array}$ & OR AJUSTADO * (IC 95\%) \\
\hline $\begin{array}{c}\text { Satisfacción } \\
\text { Modalidad }\end{array}$ & $\mathbf{0 , 0 0 1}$ & $3,125(1,60-6,09)$ & 0,329 & $1,772(0,56-5,59)$ \\
$\begin{array}{c}\text { Competencia } \\
\text { profesional }\end{array}$ & $\mathbf{0 , 0 0 1}$ & $2,999(1,60-5,61)$ & 0,874 & $0,916(0,31-218,35)$ \\
$\begin{array}{c}\text { Repercusión } \\
\text { económica }\end{array}$ & $\mathbf{0 , 0 1 9}$ & $12,744(1,52-106,54)$ & 0,161 & $7,174(0,46-112,96)$ \\
& AUC & & & $0,930(0,82-1,03)$ \\
\hline
\end{tabular}

Fuente: Elaboración propia. 
EI OR crudo (univariante) de todas las variables se interpreta como un incremento de la probabilidad de mejorar la calidad asistencial al aumentar en una unidad la variable independiente. En relación, al OR ajustado (multivariante), la única variable que permanece como significativa es la modalidad formativa. Al incrementar el valor de esta dimensión aumenta de forma significativa la probabilidad de mejorar la calidad asistencial permaneciendo el resto de las dimensiones constantes.

\section{Conclusiones}

En este trabajo de evaluación de impacto formativo se ha intentado poner a prueba un modelo evaluativo holístico, sistematizado y adaptativo. Holístico, dada su concepción global de la organización y la formación. Sistematizado, por su estructura en procesos y fases. Adaptativo, al disponer de la capacidad de reconocer las particularidades de una intervención formativa compleja, que en inicio no contemplaba la evaluación de los efectos organizativos en su formulación. La primera conclusión sería que este tipo de programas formativos supone trascender la visión de la evaluación como algo puntual y finalista. Supone un proceso continuo y como tal debe atender a las características de ser procesual, continua e integrada en el aprendizaje (Álvarez, 2001).

La modalidad formativa hace referencia a la influencia de la manera de enseñar en los efectos de la organización, en concreto en el resultado final. Cuando el análisis bivariante se realiza entre variables suma, la media de quienes prefieren la metodología learning by doing y han mejorado sus conocimientos y habilidades, aplicándolo al lugar del trabajo es superior a la que no los aplican.

Esta forma de enseñar-aprender presenta un alto grado de satisfacción individual y de éxito a la hora de asociar los conocimientos y habilidades adquiridas con la mejora de la calidad asistencial (Reinoso, 2006). La satisfacción y el aprendizaje se pueden considerar efecto inmediatos de la actuación formativa. En la muestra del estudio, la satisfacción con el aprendizaje se mantiene tras cuatro meses de haber realizado la actividad formativa. No se trata de una valoración puntual positiva del trabajo formativo realizado. Al considerar la utilidad de lo aprendido, se tiende a estar satisfecho con el proceso formativo y aplicar dichos contenidos (Granado, Puig y Aguilar, 2012). Por tanto, la modalidad learning by doing se puede considerar una estrategia formativa adecuada y relevante en la capacitación del personal sanitario.

En el análisis bivariante se observa que se da una relación directa entre el conocimiento y la aplicación del mismo. Del mismo modo, interacciona con la calidad asistencial. Esta conexión, se puede explicar, por la formación previa del profesional sanitario, basada en gran parte en el aprendizaje observacional. También, en el hecho de que la metodología learning by doing, democratiza la relación docentealumno y se da dentro de un contexto natural que ayuda a reducir las resistencias a introducir innovaciones y mejoras en el puesto de trabajo (Chacón et al., 2006). Se considera un acierto del programa el hecho de que los docentes del curso sean compañeros de trabajo. Sin duda, la figura de profesional-docente contribuye a que los contenidos aprendidos puedan ser transferidos con más facilidad al puesto de trabajo. 
La mejora de la calidad asistencial se configura como el producto final de la intervención de aprendizaje. En este caso está integrada por contenidos de práctica clínica, es decir, el aprendizaje de técnicas diagnósticas, quirúrgicas y terapéuticas. Los profesionales coinciden en valorar positivamente su experiencia formativa en este sentido, destacando de nuevo, la influencia de la modalidad formativa (learning by doing). La interrelación positiva de las variables formativas redunda en la mejora percibida de la calidad asistencial por parte del profesional-estudiante. Estos resultados permiten entender el programa focuss desde un modelo de evaluación de impacto formativo con éxito tal y como lo plantea Brikerhoff (1981) en su modelo Sucess Case Method.

No obstante, que la evaluación del impacto formativo aporte respuestas claras de mejoras individuales y organizativas, depende de una secuencia de acciones y efectos concatenados que exigen, para su implementación y medición, del compromiso responsable y transparente de las gerencias y de su extensión a lo largo de los procesos clínicos y de gestión.

\section{Limitaciones del estudio y prospectiva}

Como limitaciones de este trabajo, destaca el hecho de que la evaluación de impacto se realiza sobre un programa que se planificó e implementó sin considerar la medición de los efectos formativos sobre la organización. En otras experiencias sería interesante identificar a priori de la acción formativa, indicadores, fuentes de información y agentes evaluadores (ej. en diferentes escalas laborales y diferentes profesiones sanitarias, pacientes y gestores, etc.).

Interesa considerar en próximos proyectos, las variables que influyen en la transferencia de la mejora de la competencia profesional. Entre éstas, Burke, Holly y Hutchins (2007) proponen: características individuales de los participantes (ej. motivación, personalidad, sistema atribucional); características metodológicas de la intervención (ej. análisis de necesidades, objetivos de aprendizaje, procedimientos); las influencias del contexto laboral (las líneas estratégicas de la organización; los supervisores/mandos intermedios, la oportunidad de hacer, la cultura organizativa de la responsabilidad). La propia definición de competencia profesional puede ser revisada e incorporar además de conocimientos y habilidades, elementos actitudinales.

También es importante reseñar la elevada colinialidad que presentan las variables independientes entre sí. Sería conveniente ampliar los procedimientos de valoración a un mayor número de casos y a otras estrategias metodológicas entre las que se encuentran las técnicas cualitativas (ej. grupos de discusión, observación de campo, entrevistas en profundidad, etc.).

El sistema sanitario exige de innovación e investigación constantes. La evaluación de las competencias profesionales forma parte de las estrategias organizativas y exige de su desarrollo en el ámbito de la investigación. La formación continua es una inversión de futuro y desarrollo. Sin embargo, no se nos debería olvidar que evaluar es investigar (Gracia, 2015). 


\section{REFERENCIAS BIBLIOGRÁFICAS}

Álvarez, J. M. (2001). Evaluar para conocer, examinar para excluir. Madrid: Morata.

Bausela, E. (2004). Detección de necesidades en el proceso de planificación de un servicio de orientación psicológica en el contexto de la Universidad de León (segundo estudio piloto). XXI: Revista de Educación, 6, 171-179.

Biencinto, Ch. y Carballo, R. (2006). Determinación del impacto de la formación continua en el sector sanitario: diseño de un modelo de relaciones entre dimensiones. Revista Complutense de Educación, 17(1), 77-88.

Brinkerhoff, R. O. (1987). Achieving results from training. How to evaluate human resource development to strengthen programs and increase impact. San Francisco: Josey-Bass.

Burke, L. A., Holly M. y Hutchins, H. M. (2007). Training Transfer: An Integrative Literature Review. Human Resource Development Review, 6, 263.

Cabrera, A. F. (2003). Evaluación de la formación. Madrid: Síntesis.

Capapé, J., Susaeta, L., Pin, J. R., Gallifa, A. y García, R. (2011). El control de la eficiencia de las prácticas de recursos humanos: un análisis de la realidad de las empresas que operan en España. Documento de Investigación, 923. Madrid: IESE Business School-Universidad de Navarra.

Chacón, S., Holgado, F. P., López, J. M. y Sanduvete, S. (2006). Evaluación de la formación continua: fundamentos teóricos y herramientas metodológicas. Sevilla: Universidad de Sevilla, Secretariado de Publicaciones.

Eseryel, D. (2002). Approaches to Evaluation of Training: Theory and Practice. Educational Technology \& Society, 5(2), 93-98.

Ferrández, E. (2006). La evaluación de impacto en el Master de Formación de Formadores. Informe de Investigación del Departamento de Pedagogía Aplicada. Barcelona: Universidad Autónoma de Barcelona.

Fernández, A., Porcel, A. M., Nuviala, A., Pérez, R. J., Tamayo, J., Grao, A. y González, J. J. (2012). Estudio comparativo entre una metodología de aprendizaje tradicional respecto a una metodología de aprendizaje basada en el "learning by doing" para la consecución de competencias específicas. UPO INNOVA: Revista de Innovación Docente, I, 159-166.

Gracia, M. L. (2015). Evaluación del impacto formativo: dimensiones y modelos. Una experiencia en la Sanidad de Aragón. Tesis doctoral, Departamento de Psicología y Sociología, Universidad de Zaragoza.

Granado, C., Puig, M. y Aguilar, S. (2012). ¿Planeamos la formación continua para ser transferida? Descripción de un instrumento para el análisis. Hekademos: revista educativa digital, 12, 53-60.

Green, J., Durand, M. A., Hutchings, A. y Black, N. (2011). Modernisation as a professionalising strategy: the case of critical care in England. Journal Sociology of Health \& IIIness, 33(6), 819-836.

Guerra-López, I. (2007). Evaluación y mejora continúa: conceptos y herramientas para la medición y mejora del desempeño. Bloomington: Author House.

Kirkpatrick, D. L. (2003). Evaluación de acciones formativas. Barcelona: Gestión 2000. 
Méndez, A. (2007). Terminología pedagógica específica al enfoque por competencias: el concepto de competencia. Innovación Educativa, 17, 173-184.

Merino, M. (2007). La evaluabilidad: de instrumento de gestión a herramienta estratégica en la evaluación de políticas públicas. Papeles 7/2007 de Agencia de Evaluación y Calidad. Madrid: Ministerio de Administraciones Públicas. Agencia Estatal de Evaluación de las Políticas Públicas y la Calidad de los Servicios.

Miller, G. E. (1990). The assesment of clinical skills/competence/performance. Academic Medicine (Supplement), 65, 63-67.

Moore, C. (2009). It's Time for Measurement Strategy. Chief Learning Officer Magazine, 8(40), 40-43.

Oliver, M. F. (2002). Análisis de necesidades de formación del profesorado de educación primaria de las Illes Balears. Diseño y desarrollo de una investigación. Innovación educativa, 12, 265-274.

Onrubia, J. (2005). Evaluación y gestión eficiente en el sector público: aspectos organizativos e institucionales. Ekonomiaz, 1(60), 38-57.

Peña, I. y Hernández, F. (2007). Validez del enfoque configuracional de la DRH en el sector financiero de la economía social. En Decisiones basadas en el conocimiento y en el papel social de la empresa: XX Congreso anual de AEDEM (pp. 394-406). Madrid: Academia Europea de Dirección y Economía de la Empresa.

Pineda, P. (2000). Evaluación del impacto de la formación en las organizaciones. Revista Educar, 27, 119-133.

Reinoso, M. (2006). Desarrollo de competencias emocionales en los individuos de una organización utilizando la metodología de formación: outdoor training. Tesis doctoral, Departamento de Sociología, Universidad de Granada.

Rutty, M. G. (2007). Evaluación de impacto en la capacitación de recursos humanos. Tesis doctoral, Facultad de Ciencias Económicas, Universidad de Buenos Aires.

Soto, A. (2011). Evaluación de intervenciones sanitarias y sociales: midiendo los resultados para orientar la toma de decisiones. Revista Peruana de Medicina Experimental y Salud Pública, 28(3), 414-415.

Stufflebeam, D. L. y Shinkfield, A. J. (1987). Evaluación Sistémica: guía teórica y práctica ( $1^{\text {a }}$ reimpresión). Madrid: Paidós Ibérica.

Tejada, J. (2011). La evaluación de las competencias en contextos no formales: dispositivos e instrumentos de evaluación. Revista de Educación, 354, 731-745.

Tejada, J. y Ferrández, E. (2007). La evaluación del impacto de la formación como estrategia de mejora en las organizaciones. Revista Electrónica de Investigación Educativa, 9(2). Recuperado de http://redie.uabc.mx/vol9no2/contenido-tejada2.html.

Tyler, R. W. (1942). General statement on evaluation. Journal of Educational Research, $35,492-501$.

Ubillos, S. y Goiburu, E. (2010). Evaluación del proceso, de resultados e impacto. Ponencia presentada en Jornadas Capacitando a las ONG/SIDA: mejorando la prevención del VIH. Barcelona. 